\title{
Transient computations using the natural stress formulation for solving sharp corner flows
}

\author{
J.D. Evans ${ }^{\text {a,*, C.M. Oishi }}{ }^{\mathrm{b}}$ \\ a Department of Mathematical Sciences, University of Bath, Bath, BA2 7AY, United Kingdom \\ bepartamento de Matemática e Computação, Faculdade de Ciências e Tecnologia, Universidade Estadual Paulista "Júlio de Mesquita Filho" $19060-900$ \\ Presidente Prudente, Sao Paulo, Brazil
}

\section{A R T I C L E I N F O}

\section{Article history:}

Received 26 May 2017

Revised 24 August 2017

Accepted 30 August 2017

Available online 19 September 2017

\section{Keywords:}

Unsteady viscoelastic flows

Oldroyd-B model

Natural stress formulation

Planar contraction

Sharp corner

\begin{abstract}
A B S T R A C T
In this short communication, we analyse the potential of the natural stress formulation (NSF) (i.e. aligning the stress basis along streamlines) for computing planar flows of an Oldroyd-B fluid around sharp corners. This is the first attempt to combine the NSF into a numerical strategy for solving a transient fluid flow problem considering the momentum equation in Navier-Stokes form (the elastic stress entering as a source term) and using the constitutive equations for natural stress variables. Preliminary results of the NSF are motivating in the sense that accuracy of the numerical solution for the extra stress tensor is improved near to the sharp corner. Comparison studies among the NSF and the Cartesian stress formulation (CSF) (i.e. using a fixed Cartesian stress basis) are conducted in a typical benchmark viscoelastic fluid flow involving a sharp corner: the $4: 1$ contraction. The CSF needs a mesh approximately 10 times smaller to capture similar near singularity results to the NSF.
\end{abstract}

(c) 2017 Elsevier B.V. All rights reserved.

\section{Introduction}

Flow through a contraction is a benchmark problem in computational rheology [9], where viscoelastic fluids exhibit regions of strong shearing near the walls and uniaxial extension along the centreline. The complex flow patterns that evolve have been the subject of much interest in the literature, with attention focused on: (i) vortex behaviour, both near the re-entrant corner (socalled lip vortices) and salient corners, (ii) variation of the pressure drops across the contraction with strength of fluid elasticity (Weissenberg number), (iii) particle paths upstream of the contraction and (iv) velocity overshoots along the axis of symmetry.

The main numerical approaches to simulate this flow, have been finite-difference [21], finite-element [8] and finite-volume [2]. Combinations of the methods, e.g. hybrid finite-element finitevolume [1] as well as Lagrangian and semi-Langrangian methods $[15,22]$ are also commonly employed. However, a key feature of all numerical schemes so far employed is that they discretize the viscoelastic constitutive equations formulated using a fixed Cartesian basis for the stress. We refer to this formulation as the Cartesian stress formulation (CSF) of the constitutive equations. An alternative approach is to exploit the mathematical structure of the upper

\footnotetext{
* Corresponding author.

E-mail addresses: masjde@maths.bath.ac.uk, masjde@bath.ac.uk (J.D. Evans), oishi@fct.unesp.br (C.M. Oishi).
}

convected derivative and align the stress basis with the flow using streamlines. This formulation uses the velocity field to span the stress field, the formulation of the constitutive equations in this setting being termed the natural stress formulation (NSF).

The natural stress formulation was first used by Renardy [17], to demonstrate its ability to eliminate the downstream stress instability encountered during numerical integration around re-entrant corners [18]. Although the idea of transforming the stress tensor components to a basis aligned with streamlines for computation purposes had been recognised previously in $[3,11]$. However, the full power of the approach has not yet been exploited numerically in a mathematically systematic way for the full contraction geometry.

A key feature of the geometry is the presence of the re-entrant corner at which the velocity gradients and stress are infinite. The singularity determination for the Oldroyd-B fluid was first given by Hinch [10], with the asymptotic structure of the local solution completed through the upstream wall boundary layer by Renardy [19] and the boundary layer at the downstream wall in [16] and $[4,5]$. An important aspect of the solution analysis is that the natural stress formulation is an efficient way to transmit the necessary stress information from the upstream to downstream regions. This is explicitly calculated in [6,7] for the UCM fluid, illustrating that the necessary stress information is contained in high-order terms of the asymptotic expansions when using the Cartesian stress com- 
ponents, with the natural stress variables being able to uncouple and extract this information.

The success of the natural stress formulation near the reentrant corner singularity, both for asymptotics and numerical computation, are the motivating reasons to investigate the formulation for the full contraction geometry.

\section{Mathematical formulation}

The governing equations for the incompressible flow of a viscoelastic fluid we adopt are the continuity, momentum and Oldroyd-B constitutive equations in dimensionless form

$\nabla \cdot \mathbf{v}=0$

$\operatorname{Re}\left(\frac{\partial \mathbf{v}}{\partial t}+\mathbf{v} \cdot \nabla \mathbf{v}\right)=-\nabla p+\beta \nabla^{2} \mathbf{v}+\nabla \cdot \mathbf{T}$,

$\mathbf{T}+\mathrm{Wi}\left(\frac{\partial \mathbf{T}}{\partial t}+(\mathbf{v} \cdot \nabla) \mathbf{T}-(\nabla \mathbf{v}) \mathbf{T}-\mathbf{T}(\nabla \mathbf{v})^{T}\right)=2(1-\beta) \mathbf{D}$.

Here $\mathbf{v}$ is the velocity field, $p$ is an arbitrary isotropic pressure, $\mathbf{T}$ is the polymeric contribution to the extra-stress tensor and $\mathbf{D}=$ $\frac{1}{2}\left(\nabla \mathbf{v}+(\nabla \mathbf{v})^{T}\right)$ is the rate-of-strain tensor. The dimensionless parameters are the Reynolds number Re, Weissenberg number Wi and retardation parameter $\beta \in[0,1]$ (the dimensionless retardation time or solvent viscosity ratio) defined by

$\operatorname{Re}=\frac{\rho U L}{\eta_{s}+\eta_{p}}, \quad \mathrm{Wi}=\frac{\lambda_{1} U}{L}, \quad \beta=\frac{\eta_{s}}{\eta_{s}+\eta_{p}}$,

where $\rho$ is the density, $U$ and $L$ are characteristic length and flow speeds respectively, $\lambda_{1}$ the relaxation time, $\eta_{s}$ the solvent viscosity and $\eta_{p}$ the polymer viscosity. The above governing equations have been made non-dimensional using $L$ for the spatial variables, $U$ for the velocity scaling and $\left(\eta_{p}+\eta_{s}\right) U / L$ the pressure and stress scalings. The total stress is $\sigma=-p \mathbf{I}+\boldsymbol{\tau}$, with the extra-stress tensor $\boldsymbol{\tau}=\mathbf{T}+2 \beta \mathbf{D}$ being rheologically composed of polymer and Newtonian solvent contributions.

\subsection{Cartesian stress formulation}

Denoting by $\mathbf{i}$ and $\mathbf{j}$ the unit vectors in fixed Cartesian $x$ and $y$ directions, we have

$\mathbf{v}=u \mathbf{i}+v \mathbf{j}=(u, v)^{T}$

and

$\mathbf{T}=T_{11} \mathbf{i i}^{T}+T_{12}\left(\mathbf{i j}^{T}+\mathbf{j i}^{T}\right)+T_{22} \mathbf{j j}^{T}$.

The component form of the polymer constitutive Eq. (3) for the Cartesian extra-stresses $T_{11}, T_{12}, T_{22}$ is

$$
\begin{aligned}
T_{11} & +\operatorname{Wi}\left(\frac{\partial T_{11}}{\partial t}+u \frac{\partial T_{11}}{\partial x}+v \frac{\partial T_{11}}{\partial y}-2 \frac{\partial u}{\partial x} T_{11}-2 \frac{\partial u}{\partial y} T_{12}\right) \\
= & 2(1-\beta) \frac{\partial u}{\partial x}, \\
T_{22} & +\operatorname{Wi}\left(\frac{\partial T_{22}}{\partial t}+u \frac{\partial T_{22}}{\partial x}+v \frac{\partial T_{22}}{\partial y}-2 \frac{\partial v}{\partial y} T_{22}-2 \frac{\partial v}{\partial x} T_{12}\right) \\
= & 2(1-\beta) \frac{\partial v}{\partial y}, \\
T_{12} & +\operatorname{Wi}\left(\frac{\partial T_{12}}{\partial t}+u \frac{\partial T_{12}}{\partial x}+v \frac{\partial T_{12}}{\partial y}-\frac{\partial v}{\partial x} T_{11}-\frac{\partial u}{\partial y} T_{22}\right) \\
= & (1-\beta)\left(\frac{\partial u}{\partial y}+\frac{\partial v}{\partial x}\right) .
\end{aligned}
$$

\subsection{Natural stress formulation}

Aligning the polymer stress basis along streamlines, introduces the so called natural stress variables. We follow the construction of Renardy [17] (see also [20,23]). Introducing the configuration tensor A by

$\mathbf{T}=\frac{(1-\beta)}{\mathrm{Wi}}(\mathbf{A}-\mathbf{I})$,

the polymer constitutive Eq. (3) becomes

$\left[\frac{\partial \mathbf{A}}{\partial t}+(\mathbf{v} \cdot \nabla) \mathbf{A}-(\nabla \mathbf{v}) \mathbf{A}-\mathbf{A}(\nabla \mathbf{v})^{T}\right]+\frac{1}{\mathrm{Wi}}(\mathbf{A}-\mathbf{I})=0$.

We now express $\mathbf{A}$ in terms of the dyadic products of the velocity $\mathbf{v}$ and an orthogonal vector $\mathbf{w}$ defined as follows

$\mathbf{w}=\frac{1}{|\mathbf{v}|^{2}}(-v, u)^{T}$

so that

$\mathbf{A}=\lambda \mathbf{v v}^{T}+\mu\left(\mathbf{v} \mathbf{w}^{T}+\mathbf{w} \mathbf{v}^{T}\right)+\nu \mathbf{w} \mathbf{w}^{T}$,

where $\lambda, \mu, v$ are termed the natural stress variables. However, a detraction of this construction is that the basis vectors $\mathbf{v}, \mathbf{w}$ are degenerate when the velocity field vanishes. As such, it is convenient to use instead, unit vectors in their directions. Hence, we write

$\mathbf{A}=\hat{\lambda} \hat{\mathbf{v}}^{T}+\hat{\mu}\left(\hat{\mathbf{v}} \hat{\mathbf{w}}^{T}+\hat{\mathbf{w}} \hat{\mathbf{v}}^{T}\right)+\hat{v} \hat{\mathbf{w}} \hat{\mathbf{w}}^{T}$,

where

$\hat{\mathbf{v}}=\frac{\mathbf{v}}{|\mathbf{v}|}, \quad \hat{\mathbf{w}}=|\mathbf{v}| \mathbf{w}, \quad \hat{\lambda}=|\mathbf{v}|^{2} \lambda, \quad \hat{\mu}=\mu, \quad \hat{v}=\frac{v}{|\mathbf{v}|^{2}}$.

The scaled natural stress variables $\hat{\lambda}, \hat{\mu}, \hat{v}$ then satisfy the component equations

$\left[\frac{\partial \hat{\lambda}}{\partial t}+\frac{2 \hat{\mu}}{|\mathbf{v}|^{2}}\left(v \frac{\partial u}{\partial t}-u \frac{\partial v}{\partial t}\right)+|\mathbf{v}|^{2}(\mathbf{v} \cdot \nabla)\left(\frac{\hat{\lambda}}{|\mathbf{v}|^{2}}\right)+2 \hat{\mu}|\mathbf{v}|^{2} \nabla \cdot \mathbf{w}\right]$ $+\frac{1}{\mathrm{Wi}}(\hat{\lambda}-1)=0$,

$\left[\frac{\partial \hat{\mu}}{\partial t}+\left(\frac{\hat{\lambda}-\hat{v}}{|\mathbf{v}|^{2}}\right)\left(u \frac{\partial v}{\partial t}-v \frac{\partial u}{\partial t}\right)+(\mathbf{v} \cdot \nabla) \hat{\mu}+\hat{v}|\mathbf{v}|^{2} \nabla \cdot \mathbf{w}\right]$ $+\frac{\hat{\mu}}{\mathrm{Wi}}=0$,

$\left[\frac{\partial \hat{v}}{\partial t}+\frac{2 \hat{\mu}}{|\mathbf{v}|^{2}}\left(u \frac{\partial v}{\partial t}-v \frac{\partial u}{\partial t}\right)+\frac{1}{|\mathbf{v}|^{2}}(\mathbf{v} \cdot \nabla)\left(\hat{v}|\mathbf{v}|^{2}\right)\right]$

$+\frac{1}{\mathrm{Wi}}(\hat{v}-1)=0$

with

$$
\begin{array}{r}
|\mathbf{v}|^{2} \nabla \cdot \mathbf{w}=|\mathbf{v}|^{2}\left(\frac{\partial}{\partial x}\left(-\frac{v}{|\mathbf{v}|^{2}}\right)+\frac{\partial}{\partial y}\left(\frac{u}{|\mathbf{v}|^{2}}\right)\right) \\
=\frac{1}{|\mathbf{v}|^{2}}\left(\left(v^{2}-u^{2}\right)\left(\frac{\partial v}{\partial x}+\frac{\partial u}{\partial y}\right)+4 u v \frac{\partial u}{\partial x}\right) .
\end{array}
$$

The component form of $(8)$ is

$$
\begin{aligned}
& T_{11}=\frac{(1-\beta)}{\mathrm{Wi}}\left(-1+\frac{1}{|\mathbf{v}|^{2}}\left(\hat{\lambda} u^{2}-2 \hat{\mu} u v+\hat{v} v^{2}\right)\right), \\
& T_{12}=\frac{(1-\beta)}{\mathrm{Wi}|\mathbf{v}|^{2}}\left(\hat{\lambda} u v+\hat{\mu}\left(u^{2}-v^{2}\right)-\hat{v} u v\right), \\
& T_{22}=\frac{(1-\beta)}{\mathrm{Wi}}\left(-1+\frac{1}{|\mathbf{v}|^{2}}\left(\hat{\lambda} v^{2}+2 \hat{\mu} u v+\hat{v} u^{2}\right)\right),
\end{aligned}
$$


with the inverse relationship being

$$
\begin{aligned}
\hat{\lambda}-1 & =\frac{\mathrm{Wi}}{(1-\beta)|\mathbf{v}|^{2}}\left(u^{2} T_{11}+2 u v T_{12}+v^{2} T_{22}\right), \\
\hat{\mu} & =\frac{\mathrm{Wi}}{(1-\beta)|\mathbf{v}|^{2}}\left(-u v T_{11}+\left(u^{2}-v^{2}\right) T_{12}+u v T_{22}\right), \\
\hat{v}-1 & =\frac{\mathrm{Wi}}{(1-\beta)|\mathbf{v}|^{2}}\left(v^{2} T_{11}-2 u v T_{12}+u^{2} T_{22}\right) .
\end{aligned}
$$

These expressions link the Cartesian and natural stress components together.

\section{Numerical method}

\subsection{CSF and NSF schemes}

The first step involved in the algorithm of the schemes is the solution of the conservative Eqs. (1) and (2) which can be written as follows:

$\frac{\partial u}{\partial x}+\frac{\partial v}{\partial y}=0$

$$
\begin{aligned}
\frac{\partial u}{\partial t}-\frac{\beta}{\operatorname{Re}}\left(\frac{\partial^{2} u}{\partial x^{2}}+\frac{\partial^{2} u}{\partial y^{2}}\right)= & -\left(\frac{\partial(u u)}{\partial x}+\frac{\partial(v u)}{\partial y}\right) \\
& -\frac{1}{\operatorname{Re}} \frac{\partial p}{\partial x}+\frac{1}{\operatorname{Re}}\left(\frac{\partial T_{11}}{\partial x}+\frac{\partial T_{12}}{\partial y}\right)
\end{aligned}
$$

$$
\begin{aligned}
\frac{\partial v}{\partial t}-\frac{\beta}{\operatorname{Re}}\left(\frac{\partial^{2} v}{\partial x^{2}}+\frac{\partial^{2} v}{\partial y^{2}}\right)= & -\left(\frac{\partial(u v)}{\partial x}+\frac{\partial(v v)}{\partial y}\right) \\
& -\frac{1}{\operatorname{Re}} \frac{\partial p}{\partial y}+\frac{1}{\operatorname{Re}}\left(\frac{\partial T_{12}}{\partial x}+\frac{\partial T_{22}}{\partial y}\right)
\end{aligned}
$$

For this purpose, in the context of a finite difference Marker-AndCell (MAC) methodology, a projection scheme for solving viscoelastic problems is applied in order to uncouple velocity and pressure fields in Eqs. (16)-(18), as carefully detailed in [13]. In summary, the momentum Eqs. (17) and (18) are solved via a semi-implicit scheme maintaining the convective terms, pressure gradient and divergence of the stress tensor discretized in the $n$ time-level. After this, the projection step is applied for obtaining the final velocity and pressure fields $\left(u^{n+1}, v^{n+1}, p^{n+1}\right)$.

Once the final velocity field is computed, the constitutive equations of the CSF or NSF can be solved. In the case of the CSF, the final extra stress components $\left(T_{11}^{n+1}, T_{12}^{n+1}, T_{22}^{n+1}\right)$ are directly calculated solving Eq. (7) by an explicit scheme [13]. For the NSF, the final values for the NS variables $\left(\hat{\lambda}^{n+1}, \hat{\mu}^{n+1}, \hat{v}^{n+1}\right)$ are computed by solving Eq. (13) via an explicit discretization scheme. The relationships (14) are then used to construct the necessary Cartesian extra stress components for use in the momentum equation. Therefore, according to the choice of the scheme (CSF or NSF), at this stage of the algorithm, all variables are updated; a new computational cycle then begins and the process continues until steady state is reached.

\subsection{Initial and boundary conditions}

In order to solve Eq. (13) numerically, initial conditions for the natural stress variables $\hat{\lambda}, \hat{\mu}$ and $\hat{v}$ are required. For instance, at $t=$ 0 , based on the relations (15) and considering that the Cartesian extra-stress tensor is initialized as $\mathbf{T}=\mathbf{0}$, we have adopted $\hat{\lambda}=\hat{v}=$ 1 and $\hat{\mu}=0$.

The boundary conditions used in this work for the application of the NSF are: prescribed inflows, outflows, and rigid walls. At the inlet, the boundary conditions on the NS variables are constructed
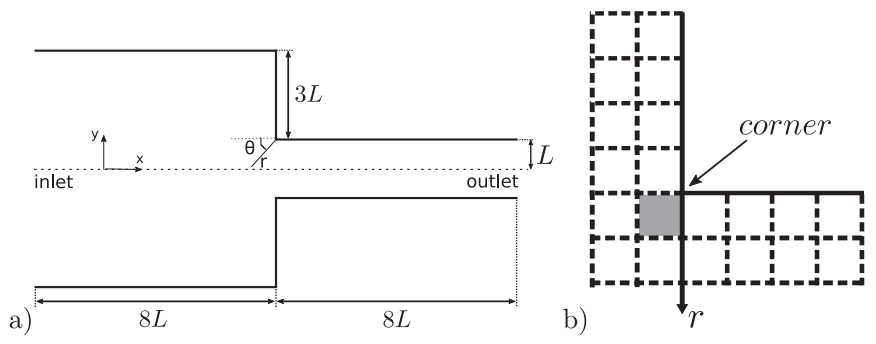

Fig. 1. a) Sketch of the $4: 1$ contraction geometry. b) Sketch of the mesh around the re-entrant corner.

as function of the extra-stress tensor. For instance, assuming fully developed flow, we have imposed:

$u=\frac{3}{4}\left(1-\frac{y^{2}}{16}\right), \quad v=0, \quad-4 \leq y \leq 4$,

and

$T_{11}=2(1-\beta) \mathrm{Wi}\left(\frac{\partial u}{\partial y}\right)^{2}$

$T_{12}=(1-\beta)\left(\frac{\partial u}{\partial y}\right)$,

$T_{22}=0$.

Substituting $v=0$ in (15), we can obtain the inlet boundary conditions for the natural stress variables:

$$
\begin{array}{ll}
\hat{\lambda} & =\frac{\mathrm{Wi}}{(1-\beta)} T_{11}+1, \quad \hat{\mu}=\frac{\mathrm{Wi}}{(1-\beta)} T_{12}, \\
\hat{v} & =\frac{\mathrm{Wi}}{(1-\beta)} T_{22}+1 .
\end{array}
$$

At an outlet, the homogeneous Neumann condition is employed for the velocity field and for the NS variables. For rigid walls, the no-slip condition is used for the velocity field. It is not necessary to impose boundary conditions for the $\hat{\lambda}, \hat{\mu}$ and $\hat{v}$ at rigid walls since the Eq. (13) are considered hyperbolic. However, in order to improve the stability behaviour of the numerical method, we have applied a linear extrapolation of the NS variables at this type of boundary.

\section{A preliminary result: planar contraction flow}

The applicability and accuracy of the NSF is now investigated in a typical benchmark problem involving a sharp re-entrant corner: the 4: 1 planar contraction flow (see Fig. 1a)). For this test, we have considered $U=1.0 \mathrm{~m} / \mathrm{s}$ for the velocity scaling while the length scaling is the half-width of the narrower channel of the contraction with $L=1.0 \mathrm{~m}$. Moreover, some simplifications are adopted:

- Reduction of the channel lengths. The main objective of this work is to present an alternative strategy for improving the computation of the non-Newtonian stress tensor around sharp corners. Therefore, the contraction domain is reduced in order to save CPU time and to give more emphasis on the numerical solution near to the re-entrant corner of the problem (see Fig. 1b)). The domain description is presented in Fig. 1a) where the inlet (upstream) and outlet (downstream) channels are constructed using a length of $8 \mathrm{~L}$. This simplification is acceptable since we are imposing fully developed velocity and stress tensor profiles at the inlet (see Section 3.2). In addition, a relatively small value for the Weissenberg number $(\mathrm{Wi}=1)$ is applied in 


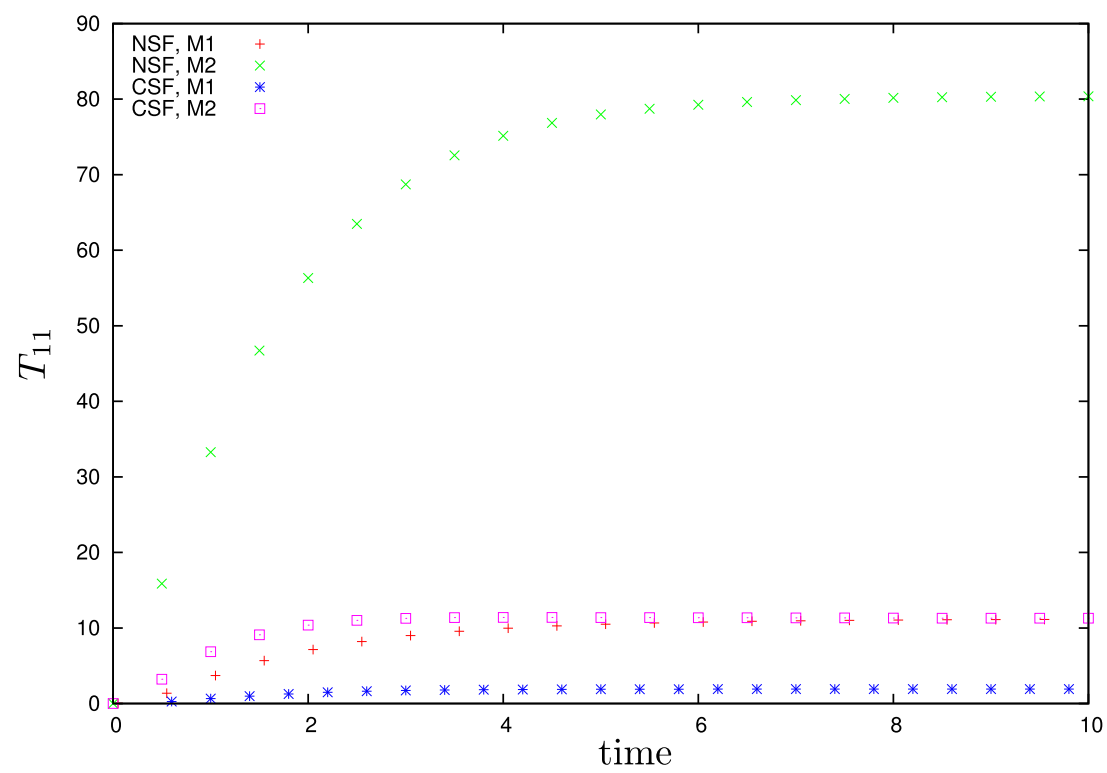

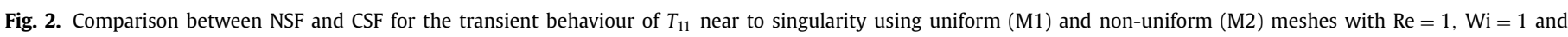
$\beta=0.5$.

Table 1

Details of the uniform and non-uniform meshes.

\begin{tabular}{lll}
\hline Mesh & Space-step & Numbers of cells \\
\hline M1 (uniform) & $\delta x=\delta y=0.05$ & 32,000 \\
M2 (non-uniform) & $\delta x_{\min }=\delta y_{\min }=0.004$ & 39,600 \\
\hline
\end{tabular}

all simulations of this Section to avoid the presence and growth of a lip vortex.

- Regularization. The basis vectors used in (10) (or in Eq. (11)) become degenerate where the velocity field vanishes, typically near solid boundaries and stagnation points. Although using the scaled variables (12) is beneficial, the transformation remains singular and care is needed in using (13). Therefore, terms are regularised in these equations in order to avoid numerical division by zero, where it may be encountered. In particular, a regularization constant tol $=10^{-6}$ is adopted in all simulations.

Further, in all simulations, we have set the following parameters: $\operatorname{Re}=1, \mathrm{Wi}=1.0$ and $\beta=0.5$. The influence of varying these parameters as well as the regularization constant tol will be carefully investigated in further work. Results for the two formulations are presented on a coarse uniform mesh M1 and a non-uniform mesh M2. Details about these meshes can be found in Table 1.

Firstly, we consider the transient behaviour of $T_{11}$ near to the geometric singularity of the contraction, specifically, in the center of the shaded cell shown in Fig. 1b). Fig. 2 shows that the NSF gives considerably larger $T_{11}$ values than the CSF in the region of the sharp corner for both meshes. The growth of the extra stress tensor when $r \rightarrow 0$, evident in Fig. 2 for the mesh M2, is expected from the asymptotic analysis which indicates that $T_{11} \propto r^{-\frac{2}{3}}$. It is worth remarking in Fig. 2 that the value of $T_{11}$ produced by the NSF in the coarse mesh is similar to the value reached by the CSF in a non-uniform mesh (approximately 10 times smaller).

In Fig. 3, we have depicted the asymptotic behaviour of the properties considering results along of the $r$ line (vertical direction) illustrated in Fig. 1b) i.e. $\theta=\pi / 2$ in Fig. 1a). We have chosen this direction consistent with the literature for ease of compari- son of the results (e.g. $[1,2,12,14])$. The results in Fig. 3 for the CSF scheme on the non-uniform mesh agree very well with those presented in $[2,14]$ for comparable mesh spacing. From these figures, we observe a significant improvement of the numerical solutions using the NSF. As we can see in Fig. 3a), even with the application of the NSF on a coarse mesh, the values of $\log \left(\left|T_{11}\right|\right)$ for the nearest three points of the corner are fitted with the slope expected for the stress. For the other stress components, the numerical solutions obtained by the NSF are in good agreement with the asymptotic analysis for $r \rightarrow 0$. In particular, it can be observed that both formulations produce similar results for the velocity field. In all figures, we have included the expected slope for the extra stress tensor and the velocity field according to asymptotic analyses of $[5,10]$. It is worth mentioning that away from the singularity, numerical results for both formulations are very similar.

\section{Discussion}

In this work, we have numerically investigated an alternative method for solving sharp corner flows involving the Oldroyd-B model. The mathematical formulation is based on a dyadic decomposition of the conformation tensor resulting in the natural stress formulation originally proposed by Renardy [17].

The main contributions of this short communication can be summarized as follows:

- The NSF equations relevant to modelling two dimensional unsteady flows have been presented and used to numerically solve a complex flow;

- The numerical results indicate that the NSF gives an effective formulation for capturing the expected behaviour of the stress near to the singularity even in the application of coarse meshes.

In the extension of this work, further details regarding the construction of the numerical method for the NSF will be provided along with the possibility of devising a hybrid scheme in which the CSF is used at solid boundaries. 
a)
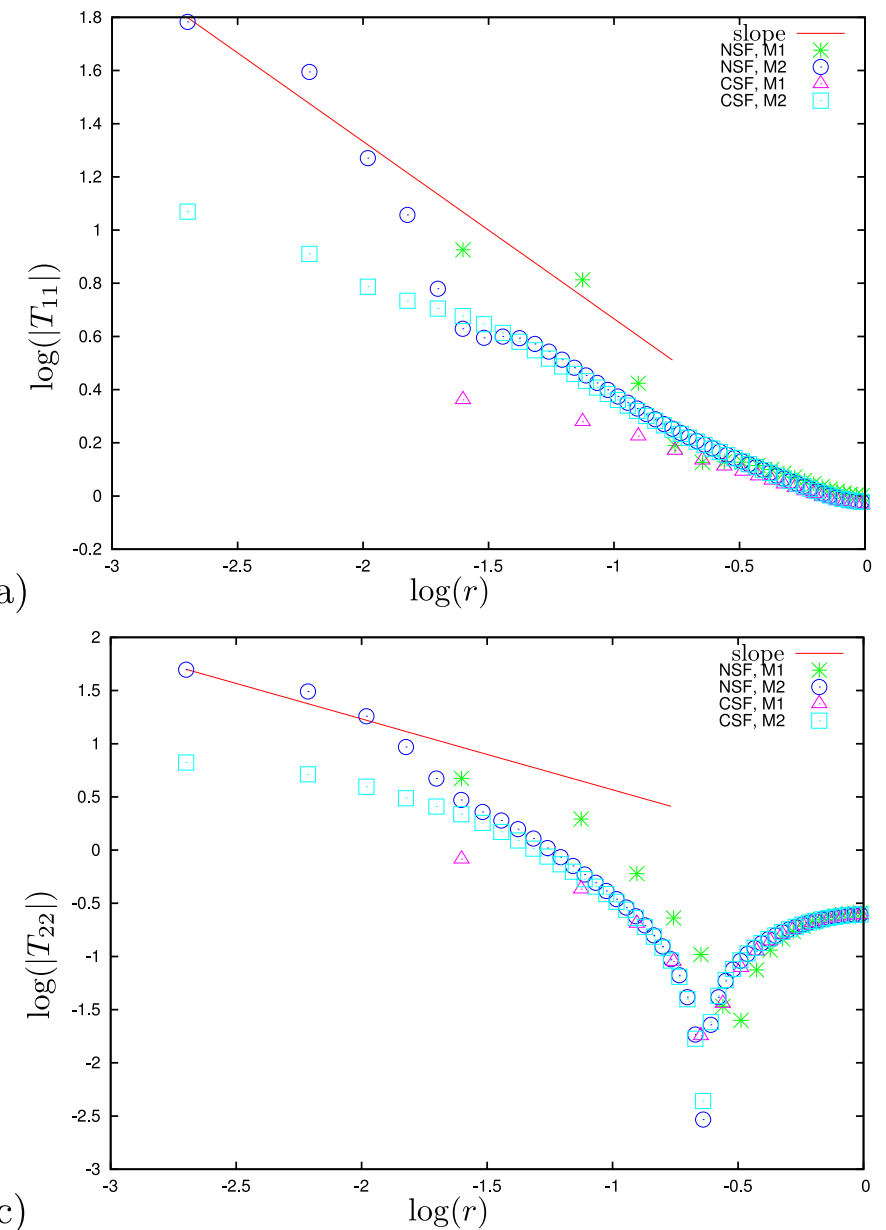

b)

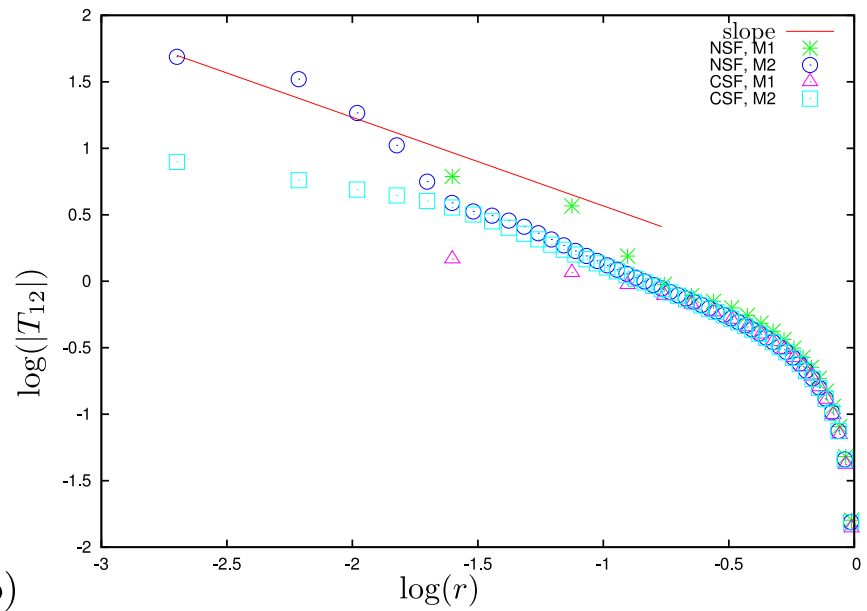

d)

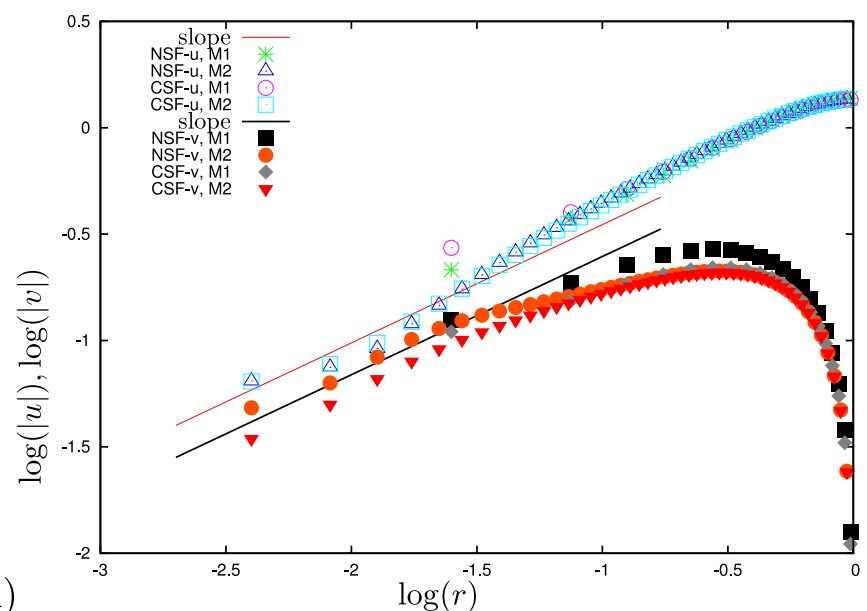

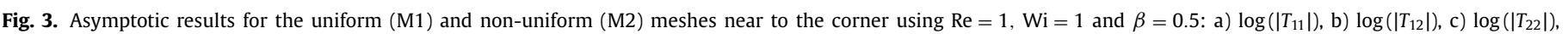
and d) $\log (|u|)$ and $\log (|v|)$.

\section{Acknowledgements}

The authors would like to thank the financial support given by SPRINT/FAPESP grant no. 2015/50094-7 and The Royal Society Newton International Exchanges grant no. 2015/NI150225. C.M. Oishi acknowledges CNPq (Conselho Nacional de Desenvolvimento Científico e Tecnologico), Grant 307459/2016-0, and FAPESP, Grant 2013/07375-0. The authors would like to thank Hugo L. França for his support and assistance with the non-uniform mesh generation.

\section{References}

[1] M. Aboubacar, H. Matallah, M.F. Webster, Highly elastic solutions for Oldroyd$\mathrm{B}$ and Phan-Thien/Tanner fluids with a finite volume/element method: planar contraction flows, J. Non-Newtonian Fluid Mech. 103 (2002) 65-103.

[2] M.A. Alves, P.J. Oliveira, F.T. Pinho, Benchmark solutions for the flow of Oldroyd-B and PTT fluids in planar contractions, J. Non-Newtonian Fluid Mech. 110 (2003) 45-75.

[3] S. Dupont, J.M. Marchal, M.J. Crochet, Finite element simulation of viscoelastic fluids of the integral type, J. Non-Newtonian Fluid Mech. 17 (1985) 157-183.

[4] J.D. Evans, Re-entrant corner flows of the upper convected Maxwell fluid, Proc. Roy. Soc. A 461 (2005) 117-142.

[5] J.D. Evans, Re-entrant corner flows of Oldroyd-B fluids, Proc. Roy. Soc. A 461 (2005) 2573-2603.

[6] J.D. Evans, Re-entrant corner flows of UCM fluids: the Cartesian stress basis, J. Non-Newtonian Fluid Mech. 150 (2008) 116-138.

[7] J.D. Evans, Re-entrant corner flows of UCM fluids: the natural stress basis, J. Non-Newtonian Fluid Mech. 150 (2008) 139-153.

[8] R. Guènette, M. Fortin, A new mixed finite element method for computing viscoelastic flows, J. Non-Newtonian Fluid Mech. 60 (1995) 27-52.

[9] O. Hassager, Working group on numerical techniques, in: proceedings of the fifth international workshop on numerical methods in non-Newtonian flows, lake arrowhead, USA, J. Non-Newtonian Fluid Mech. 29 (1988) 2-5.
[10] E.J. Hinch, The flow of an Oldroyd fluid around a sharp corner, J. NonNewtonian Fluid Mech. 50 (1993) 161-171.

[11] R.A. Keiller, Entry-flow calculations for the Oldroyd-B and FENE equations, J. Non-Newtonian Fluid Mech. 46 (1993) 143-178.

[12] J.M. Kim, C. Kim, J.H. Kim, C. Chung, K.H. Ahn, S.J. Lee, High-resolution finite element simulation of 4:1 planar contraction flow of viscoelastic fluid, J Non-Newtonian Fluid Mech. 129 (2005) 23-37.

[13] C.M. Oishi, F.P. Martins, M.F. Tomé, J.A. Cuminato, S. McKee, Numerical solution of the extended pom-pom model for viscoelastic free surface flows, J. Non-Newtonian Fluid Mech. 166 (2011) 165-179.

[14] F. Pimenta, M.A. Alves, Stabilization of an open-source finite-volume solver for viscoelastic fluid flows, J. Non-Newtonian Fluid Mech. 239 (2017) 85-104.

[15] T.N. Phillips, A.J. Williams, Viscoelastic flow through a planar contraction using a semi-Lagrangian finite volume method, J. Non-Newtonian Fluid Mech. 87 (1999) 215-246.

[16] J.M. Rallison, E.J. Hinch, The flow of an Oldroyd fluid past a reentrant corner: the downstream boundary layer, J. Non-Newtonian Fluid Mech. 116 (2004) 141-162.

[17] M. Renardy, How to integrate the upper convected Maxwell (UCM) stresses near a singularity (and maybe elsewhere, too), J. Non-Newtonian Fluid Mech. 52 (1994) 91-95.

[18] M. Renardy, The stresses of an upper convected Maxwell fluid in a Newtonian velocity field near a re-entrant corner, J. Non-Newtonian Fluid Mech. 50 (1993) $127-134$.

[19] M. Renardy, A matched solution for corner flow of the upper convected Maxwell fluid, J. Non-Newtonian Fluid Mech. 58 (1995) 83-89.

[20] M. Renardy, The high Weissenberg number limit of the UCM model and the Euler equations, J. Non-Newtonian Fluid Mech. 69 (1997) 293-301.

[21] D. Trebotich, P. Colella, G.H. Miller, A stable and convergent scheme for viscoelastic flow in contraction channels, J. Comput. Phys 205 (2005) 315-342.

[22] P. Wapperom, R. Keunings, V. Legat, The backward-tracking Lagrangian particle method for transient viscoelastic flows, J. Non-Newtonian Fluid Mech. 91 (2000) 273-295.

[23] P. Wapperom, M. Renardy, Numerical prediction of the boundary layers in the flow around a cylinder using a fixed velocity field, J. Non-Newtonian Fluid Mech. 125 (2005) 35-48. 